\title{
Event-plane correlators
}

\author{
Rajeev S. Bhalerao, ${ }^{1}$ Jean-Yves Ollitrault, ${ }^{2}$ and Subrata Pal ${ }^{3}$ \\ ${ }^{1}$ Department of Theoretical Physics, Tata Institute of Fundamental Research, Homi Bhabha Road, Mumbai 400005, India \\ ${ }^{2}$ CNRS, URA2306, IPhT, Institut de physique theorique de Saclay, F-91191 Gif-sur-Yvette, France \\ ${ }^{3}$ Department of Nuclear and Atomic Physics, Tata Institute of \\ Fundamental Research, Homi Bhabha Road, Mumbai 400005, India
}

(Dated: September 11, 2018)

\begin{abstract}
Correlators between event planes of different harmonics in relativistic heavy-ion collisions have the potential to provide crucial information on the initial state of the matter formed in these collisions. We present a new procedure for analyzing such correlators, which is less demanding in terms of detector acceptance than the one used recently by the ATLAS collaboration to measure various two-plane and three-plane correlators in $\mathrm{Pb}-\mathrm{Pb}$ collisions at LHC. It can also be used unambiguously for quantitative comparison between theory and data. We use this procedure to carry out realistic simulations within the transport model AMPT. Our theoretical results are in excellent agreement with the ATLAS data, in contrast with previous hydrodynamic calculations which only achieved qualitative agreement. We present predictions for new correlators, in particular four-plane correlators, which can easily be analyzed with our new method.
\end{abstract}

PACS numbers: $25.75 . \mathrm{Ld}, 24.10 . \mathrm{Nz}$

Introduction.-High-energy heavy-ion collision experiments at the Relativistic Heavy-Ion Collider (RHIC), BNL and the Large Hadron Collider (LHC), CERN have firmly established the formation of strongly interacting matter which exhibits a strong collective flow [1 3]. This not only suggests that the matter formed is close to local thermal equilibrium but also provides a window to the initial state of the fireball immediately after the collision. Collective flow in the plane transverse to the beam axis is typically measured in terms of two-particle angular correlations [4 7] and its small anisotropies captured in its Fourier harmonics [8-11]. Recently, a new tool, namely correlations among event planes corresponding to different harmonics [12 14], is emerging with a promise to throw additional light on the initial-state phenomena.

Pair correlations (i.e., the single-particle anisotropic flow $v_{n}$ extracted from two-particle correlations) are reasonably well understood [8]. Event-plane correlators represent higher-order correlations, involving at least three particles. Such higher-order correlations thus open a new direction in heavy-ion physics, much in the same way as studies of non-Gaussian fluctuations [15] in the early Universe. They bring in a large number of new observables which provide new, detailed insight into the hydrodynamic response and on the initial stage, and will significantly improve our understanding of heavy-ion collisions.

The ATLAS collaboration [14 has recently released preliminary measurements of eight two-plane correlators (e.g., the correlation between the second and fourth harmonics) and six three-plane correlators (e.g., the mixed correlation between second, third and fifth harmonics) in $\mathrm{Pb}-\mathrm{Pb}$ collisions at $\sqrt{s_{N N}}=2.76 \mathrm{TeV}$, as a function of the centrality of the collision. These correlators are qualitatively understood within event-by-event hydrodynamics [16] and provide new insight [17] into the inter- play between the linear and nonlinear [18] hydrodynamic response [19, 20] to the initial density profile.

The ATLAS analysis is very demanding in terms of detector acceptance: each event plane is determined in a separate pseudorapidity window; windows must be pairwise separated by gaps in order to suppress nonflow correlations; finally, each window must be wide enough to achieve a significant resolution in every Fourier harmonic up to order six.

In this article, we show that the analysis can be done more simply, and that even three- and four-plane correlators can be safely analyzed with just two symmetric pseudorapidity windows, in the same way as two-plane correlators. The analysis can thus be performed by other experiments with smaller detector acceptance. We also explain in detail how to generalize the scalar-product method used earlier for two-particle flow analysis [21], to mixed correlations, so as to eliminate the ambiguity brought about by the event-plane method [22].

We carry out realistic simulations within a multiphase transport (AMPT) model [23]. Results are for the first time in quantitative agreement with the ATLAS measurements. In addition, we present predictions for several new correlators, in particular four-plane correlators.

Method.-The azimuthal distribution of outgoing particles is decomposed in Fourier harmonics in each collision event. The flow vector in harmonic $n$ is defined as 24],

$$
Q_{n}=\left|Q_{n}\right| e^{i n \Psi_{n}} \equiv \frac{1}{N} \sum_{j} e^{i n \phi_{j}},
$$

where we have used a complex notation and the sum runs over $N$ particles seen in a reference detector, and $\phi_{j}$ are their azimuthal angles. $\Psi_{n}$ is dubbed the event-plane angle in harmonic $n$. Note that $Q_{-n}=Q_{n}^{*}$. 
The idea of ATLAS [14] is to measure angular correlations between different harmonics, that is, between $\Psi_{n}$ and $\Psi_{m}$ with $n \neq m$. We take as an illustration the correlation between the 2 nd and 4 th harmonic planes $\Psi_{2}$ and $\Psi_{4}$. In order to ensure that the measured correlation is due to collective flow [25], one measures $\Psi_{4}$ and $\Psi_{2}$ in different parts of the detector ("subevents") $A$ and $B$ separated by a gap in pseudorapidity (i.e., polar angle). The simplest observable one can think of is $\left\langle\cos \left(4\left(\Psi_{4 A}-\Psi_{2 B}\right)\right)\right\rangle$. Since, however, $\Psi_{4 A}$ and $\Psi_{2 B}$ have statistical fluctuations due to the finite number of particles in each subevent, one corrects for these statistical fluctuations by a so-called "resolution" correction 24]. The quantity measured by ATLAS can be written explicitly as

$$
\left\langle\cos 4\left(\Phi_{2}-\Phi_{4}\right)\right\rangle \equiv \frac{\left\langle\frac{Q_{2 A}^{2}}{\left|Q_{2 A}^{2}\right|} \frac{Q_{4 B}^{*}}{\left|Q_{4 B}\right|}\right\rangle}{\sqrt{\left\langle\frac{Q_{4 A}}{Q_{4 A} \mid} \frac{Q_{4 B}^{*}}{\mid Q_{4 B}}\right|} \sqrt{\left\langle\frac{Q_{2 A}^{2}}{\left|Q_{2 A}^{2}\right|} \mid \frac{Q_{2 B}^{* 2}}{Q_{2 B}^{2} \mid}\right\rangle}},
$$

where we have used the same notation as ATLAS on the left-hand side, and angular brackets on the right-hand side denote the real part of the average over events. The numerator is $\left\langle\cos \left(4\left(\Psi_{4 A}-\Psi_{2 B}\right)\right)\right\rangle$ and the denominator is the resolution correction [26].

As shown in 22], Eq. (22) represents an ambiguous measurement, in the sense that the denominator does not properly correct for the resolution when flow fluctuations are present [27, 28]. If one repeats the analysis with a lower resolution (typically, smaller subevents $A$ and $B)$, both the numerator and the denominator decrease, but the denominator decreases faster than the numerator, resulting in an increase of the signal. The difference between the low-resolution limit and the high-resolution limit can be as large as $50 \%$ for the above correlation [22]. In practice, experimental values are likely to be close to the low-resolution limit, due to the poor resolution in higher harmonics. However, the value calculated in hydrodynamics [16, 17] is the high-resolution limit, so that a quantitative comparison between theory and data is impossible at present.

These ambiguities can easily be removed. One simply replaces Eq. (2) with [22]:

$$
c\{2,2,-4\} \equiv \frac{\left\langle Q_{2 A}^{2} Q_{4 B}^{*}\right\rangle}{\sqrt{\left\langle Q_{4 A} Q_{4 B}^{*}\right\rangle} \sqrt{\left\langle Q_{2 A}^{2} Q_{2 B}^{* 2}\right\rangle}},
$$

where the notation on the left-hand side [12] expresses that the numerator involves two particles in harmonic 2 (i.e., two factors of $Q_{2}$ ) and one particle in harmonic -4 (one factor of $Q_{4}^{*}$ ). Equations (2) and (3) are referred to as "event-plane" (EP) method and "scalar product" (SP) method [21], respectively.

The only difference is that the scalar-product method retains the information on the length of $Q_{n}$. We now explain why this simple modification ensures that the right-hand side of Eq. (3) is a well-behaved flow observable, unlike (2). The flow picture is a model in which particles are emitted randomly and independently according to some underlying probability distribution in each event [29]. The azimuthal probability distribution is written as

$$
P(\phi)=\frac{1}{2 \pi} \sum_{n=-\infty}^{+\infty} v_{n} e^{i n \Phi_{n}} e^{-i n \phi}
$$

where $v_{n}$ is the anisotropic flow in harmonic $n$ and $\Phi_{n}$ the corresponding reference direction [29, 30] (we use the convention $v_{0}=1$ ). The average over events can be performed in two steps, by first averaging over events with the same $P(\phi)$, then averaging over values of $v_{n}$ and $\Phi_{n}[22]$ :

$$
\langle\ldots\rangle=\left\langle\langle\ldots\rangle_{\mid v_{n}}\right\rangle .
$$

Equations (11) and (4) yield $\left\langle Q_{n}\right\rangle_{\mid v_{n}}=v_{n} e^{i n \Phi_{n}}$. Now, the crucial point is that Eq. (3) only involves products of $Q_{n}$ vectors. Since particles are emitted independently, the expectation value of a product is the product of expectation values, therefore

$$
\begin{aligned}
\left\langle Q_{2 A}^{2} Q_{4 B}^{*}\right\rangle_{\mid v_{n}} & =\left(v_{2}\right)^{2} v_{4} e^{4 i\left(\Phi_{2}-\Phi_{4}\right)}, \\
\left\langle Q_{4 A} Q_{4 B}^{*}\right\rangle_{\mid v_{n}} & =\left(v_{4}\right)^{2} \\
\left\langle Q_{2 A}^{2} Q_{2 B}^{* 2}\right\rangle_{\mid v_{n}} & =\left(v_{2}\right)^{4}
\end{aligned}
$$

These equations show that each of the quantities entering Eq. (3) is a well-defined flow observable.

We now explain why we choose to measure the particular combination in the right-hand side of Eq. (3). The three observables in Eq. (6) are sensitive to the magnitudes of $v_{2}$ and $v_{4}$. By taking the ratio in Eq. (3), one singles out the angular correlation, in the sense that if one multiplies $v_{2}$ and/or $v_{4}$ by a constant, the right-hand side of Eq. (3) is unchanged. We therefore call this quantity an "event-plane correlator", even though it involves the statistics of $v_{2}$ and $v_{4}$, and not just the underlying directions $\Phi_{2}$ and $\Phi_{4}$. Equations (5) and (6) predict that the right-hand side of Eq. (3) should lie between -1 and 1 by the Cauchy-Schwarz inequality [31].

It can be shown that Eq. (3) coincides with Eq. (2) in the limit of low resolution [22] but, as shown above, it is independent of the experimental resolution, unlike Eq. (2), and thus yields an unambiguous measurement. The only price to pay for the loss of ambiguity is a larger statistical error.

Note that the two-plane correlator defined by Eq. (3) actually involves a three-particle correlation [32]. This is easily seen by inserting the definition of $Q_{n}$, Eq. (1), into the numerator of Eq. (3). One obtains

$$
Q_{2 A}^{2} Q_{4 B}^{*}=\frac{1}{N_{A}^{2} N_{B}} \sum_{j \in A} \sum_{k \in A} \sum_{l \in B} e^{2 i \phi_{j}+2 i \phi_{k}-4 i \phi_{l}}
$$


that is, an average of $e^{2 i \phi_{j}+2 i \phi_{k}-4 i \phi_{l}}$ over all triplets of particles.

In order to guarantee that correlations are dominated by flow, one should in principle implement pseudorapidity gaps between all particles. In Eq. (7), however, there is no pseudorapidity gap between $j$ and $k$, and there are even self-correlation terms $j=k$. Therefore, the procedure followed by ATLAS does not, strictly speaking, avoid self correlations.

We now explain why the contribution of nonflow correlations and self-correlations to Eq. (77) is small, thus justifying the procedure. It is generally accepted that long-range correlations are dominated by flow [25] or, equivalently, that nonflow correlations are short range (with the exception of global momentum conservation, which only contributes to the first Fourier harmonic [33]). Short-range correlations are effects which correlate a particle with its neighbors with some probability, so that such correlations are typically a small fraction of selfcorrelations [34]. We thus evaluate the contribution of self-correlations to Eq. (7). The thumb rule is that each factor of $e^{i n \phi}$ gives a contribution of order $v_{n}$. Thus the right-hand side of Eq. (7) is typically of order $v_{4}\left(v_{2}\right)^{2}$. Self-correlations terms $j=k$ give a contribution of order $\left(v_{4}\right)^{2} / N_{A}$. Since $v_{4} \sim\left(v_{2}\right)^{2}$ [35], the relative magnitude of self-correlations is of order $1 / N_{A}$, which is small. Note that self-correlations can also be removed explicitly if particles are seen individually 36].

The discussion can be repeated for the three-plane correlation between harmonics 2, 3 and 5. ATLAS measures the three harmonics in three separate subevents $A, B$ and $C$. We now show how the analysis can be done with just two subevents $A$ and $B$. In close analogy with (3), we define

$$
c\{2,3,-5\} \equiv \frac{\left\langle Q_{2 A} Q_{3 A} Q_{5 B}^{*}\right\rangle}{\sqrt{\left\langle Q_{2 A} Q_{2 B}^{*}\right\rangle\left\langle Q_{3 A} Q_{3 B}^{*}\right\rangle\left\langle Q_{5 A} Q_{5 B}^{*}\right\rangle}},
$$

where the numerator is a mixed correlation between harmonics 2, 3 and 5, and the denominator the corresponding resolution correction in each harmonic. We now explain why nonflow effects are small, even though the 2nd and 3rd harmonic are measured in the same subevent $A$. We decompose the numerator of Eq. (8) as

$$
Q_{2 A} Q_{3 A} Q_{5 B}^{*}=\frac{1}{N_{A}^{2} N_{B}} \sum_{j \in A} \sum_{k \in A} \sum_{l \in B} e^{2 i \phi_{j}+3 i \phi_{k}-5 i \phi_{l}} .
$$

Flow typically gives a contribution of order $v_{2} v_{3} v_{5}$. Self correlations are terms $j=k$, which give a contribution of order $\left(v_{5}\right)^{2} / N_{A}$. Since $v_{5} \sim v_{2} v_{3}$ [20], the relative order of self-correlations (and therefore of nonflow correlations) is $1 / N_{A}$, exactly as for the two-plane correlator $c\{2,2,-4\}$ considered above. Therefore this three-plane correlator does not require three separate subevents.

This discussion can be easily generalized to all other correlations. The general prescription for the pseudorapidity gap is that all the positive harmonics (i.e., all factors of $Q_{n}$ ) should go on one side (e.g., $A$ ), and all negative harmonics (all factors of $Q_{n}^{*}$ ) on the other side $(B)$. In this case, self-correlation terms are of relative order $v_{n+p} /\left(N v_{n} v_{p}\right) \sim 1 / N$, and nonflow effects can be safely neglected. Therefore all three-plane correlators measured by ATLAS can be analyzed with a single pseudorapidity gap. The analysis can be extended to fourplane correlators and higher.

If $k_{n}$ denotes the number of particles in harmonic $n$, the correlator can be generally written as

$$
c\{\cdots, \underbrace{n, n, n}_{k_{n}}, \cdots\} \equiv \frac{\left\langle\prod_{n>0}\left(Q_{n A}\right)^{k_{n}} \prod_{n<0}\left(Q_{n B}\right)^{k_{n}}\right\rangle}{\sqrt{\prod_{n}\left\langle\left(Q_{n A}\right)^{k_{n}}\left(Q_{n B}^{*}\right)^{k_{n}}\right\rangle}} .
$$

Azimuthal symmetry requires $\sum_{n} n k_{n}=0$ [12]. As an example, we write explicitly the formula for the lowestorder four-plane correlator [37]:

$$
\begin{aligned}
& c\{2,-3,-4,5\} \equiv \\
& \frac{\left\langle Q_{2 A} Q_{5 A} Q_{3 B}^{*} Q_{4 B}^{*}\right\rangle}{\sqrt{\left\langle Q_{2 A} Q_{2 B}^{*}\right\rangle\left\langle Q_{3 A} Q_{3 B}^{*}\right\rangle\left\langle Q_{4 A} Q_{4 B}^{*}\right\rangle\left\langle Q_{5 A} Q_{5 B}^{*}\right\rangle}},
\end{aligned}
$$

for which we give the first quantitative prediction below. While two-plane correlations thus defined all lie between -1 and +1 by the Cauchy-Schwarz inequality, there is no such restriction for three- and four-plane correlators. We shall give below an example of a four-plane correlator exceeding unity in AMPT calculations.

Table I lists the fourteen correlations studied by ATLAS, as well as six additional correlations predicted for the first time in this paper. For clarity, each correlation is written using the same notation as ATLAS (left column). The next columns list the number of particles in harmonics 2 to 6 . In our notation, the first two correlators are written as $c\{2,2,-4\}$ and $c\{2,2,2,2,-4,-4\}$, respectively. Table【shows that the two-plane correlators measured by ATLAS actually involve three to nine particles - never just two - yet they can safely be analyzed with a single pseudorapidity gap, and so can three- and four-plane correlators. Table \also shows that more particles are allowed in lower harmonics where the resolution is better.

Results.-Calculations are performed using the AMPT model 23] that consists of four main components: initial conditions based on Glauber model, parton cascade, hadronization, and hadron scattering. We employ the version of AMPT with string melting and parton coalescence for hadronization that describes better the collective behavior in heavy-ion collisions at RHIC and LHC $[23,38,39]$. Flow is mostly produced in this model by elastic scatterings in the partonic phase. In addition, the model contains resonance decays and thus includes nontrivial nonflow effects. The implementation used here is the same as in Ref. [39], and uses initial conditions from 
TABLE I. List of two-, three- and four-plane correlators. For each correlator, the number in column $n$ indicates the number $k_{n}$ of particles involved in harmonic $n$. Asterisks indicate that the particles actually go into harmonic $-n$ instead of $n$. The last column is the order of the correlation, i.e., the number of particles $\sum_{n} k_{n}$. The first fourteen lines correspond to the correlators measured by ATLAS [14] (correlators in italics are not studied in this paper), and the next six lines to new correlators predicted in this paper.

\begin{tabular}{|l|l|l|l|l|l|l|}
\hline & 2 & 3 & 4 & 5 & 6 & Order \\
\hline $\cos \left(4\left(\Phi_{2}-\Phi_{4}\right)\right)$ & 2 & & $1^{*}$ & & & 3 \\
$\cos \left(8\left(\Phi_{2}-\Phi_{4}\right)\right)$ & 4 & & $2^{*}$ & & & 6 \\
$\cos \left(12\left(\Phi_{2}-\Phi_{4}\right)\right)$ & 6 & & $3^{*}$ & & & 9 \\
$\cos \left(6\left(\Phi_{2}-\Phi_{3}\right)\right)$ & 3 & $2^{*}$ & & & & 5 \\
$\cos \left(6\left(\Phi_{2}-\Phi_{6}\right)\right)$ & 3 & & & & $1^{*}$ & 4 \\
$\cos \left(6\left(\Phi_{3}-\Phi_{6}\right)\right)$ & & & & $1^{*}$ & 3 \\
$\cos \left(12\left(\Phi_{3}-\Phi_{4}\right)\right)$ & 5 & & & $2^{*}$ & & 7 \\
$\cos \left(10\left(\Phi_{2}-\Phi_{5}\right)\right)$ & 1 & 1 & & $1^{*}$ & & 3 \\
$\cos \left(2 \Phi_{2}+3 \Phi_{3}-5 \Phi_{5}\right)$ & 1 & & 1 & & $1^{*}$ & 3 \\
$\cos \left(2 \Phi_{2}+4 \Phi_{4}-6 \Phi_{6}\right)$ & 1 & $2^{*}$ & 1 & & & 4 \\
$\cos \left(2 \Phi_{2}-6 \Phi_{3}+4 \Phi_{4}\right)$ & 4 & $1^{*}$ & & $1^{*}$ & & 6 \\
$\cos \left(8 \Phi_{2}-3 \Phi_{3}-5 \Phi_{5}\right)$ & 5 & & $1^{*}$ & & $1^{*}$ & 7 \\
$\cos \left(10 \Phi_{2}-4 \Phi_{4}-6 \Phi_{6}\right)$ & 5 & $2^{*}$ & $1^{*}$ & & & 8 \\
$\cos \left(10 \Phi_{2}-6 \Phi_{3}-4 \Phi_{4}\right)$ & 1 & 2 & $2^{*}$ & & & 5 \\
\hline $\cos \left(2 \Phi_{2}+6 \Phi_{3}-8 \Phi_{4}\right)$ & & 1 & $2^{*}$ & 1 & & 4 \\
$\cos \left(3 \Phi_{3}-8 \Phi_{4}+5 \Phi_{5}\right)$ & & 3 & $1^{*}$ & $1^{*}$ & & 5 \\
$\cos \left(9 \Phi_{3}-4 \Phi_{4}-5 \Phi_{5}\right)$ & 1 & $1^{*}$ & $1^{*}$ & 1 & & 4 \\
$\cos \left(2 \Phi_{2}-3 \Phi_{3}-4 \Phi_{4}+5 \Phi_{5}\right.$ & & & \\
$\cos \left(4 \Phi_{2}-3 \Phi_{3}+4 \Phi_{4}-5 \Phi_{5}\right)$ & 2 & $1^{*}$ & 1 & $1^{*}$ & & 5 \\
$\cos \left(6 \Phi_{2}+3 \Phi_{3}-4 \Phi_{4}-5 \Phi_{5}\right)$ & 3 & 1 & $1^{*}$ & $1^{*}$ & & 6 \\
\hline
\end{tabular}

the HIJING 2.0 model [40]. We have checked that it reproduces LHC data for anisotropic flow $\left(v_{2}\right.$ to $\left.v_{6}\right)$ at all centralities [38, 39, 41].

AMPT simulation events can be analyzed with the same methods as actual experimental events. However, in AMPT, the energy-momentum information of all finalstate particles in an event is available, whereas ATLAS only detects charged particles with $p_{T}>0.5 \mathrm{GeV} / \mathrm{c}$. We, therefore, use all particles in our analysis which results in a better resolution.

As explained above, our analysis of event-plane correlations uses two subevents $A$ and $B$, for which we use two symmetric pseudorapidity intervals $-4.8<\eta<-0.4$ and $0.4<\eta<4.8$, very similar to those by ATLAS for two-plane correlators. Each correlator is analyzed using both scalar-product (SP) method (Eq. (10)) and the event-plane (EP) method (in which one replaces $Q_{n}$ by $Q_{n} /\left|Q_{n}\right|$ everywhere in Eq. (10)). Recall that the EP result typically increases (in absolute magnitude) as the resolution decreases, while the SP result is the limit of low resolution. We thus expect that SP is larger than EP. If the model is valid, the experimental result (which is obtained with the EP method, but a lower resolution)
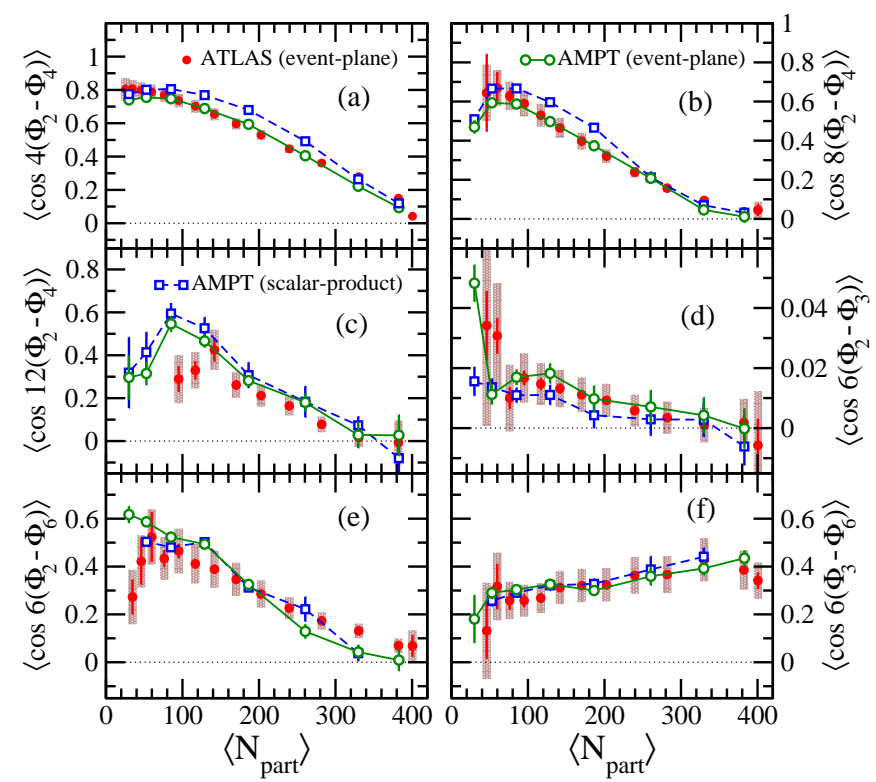

FIG. 1. (Color online) Two-plane correlators in the eventplane (open circles) and scalar-product (open squares) methods as a function of the number of participants in $\mathrm{Pb}-\mathrm{Pb}$ collisions at $\sqrt{s_{N N}}=2.76 \mathrm{TeV}$ in the AMPT model as compared to the ATLAS data [14] for the event-plane method (solid circles). We keep the notation of ATLAS $\langle\cos (\cdots)\rangle$ for simplicity, even though the measured quantities are not pure event-plane correlators.

should lie somewhere between the two AMPT predictions.

Figure 1 displays the results for two-plane correlators. Figures 1(a) and1(b), which have the smallest error bars, show that the SP method gives larger results than the EP method, as expected [22]. The difference is in the range $(10-15) \%$. EP results are in perfect agreement with data. This is in contrast with EP calculations in hydrodynamics, which are clearly below data [16, 17].
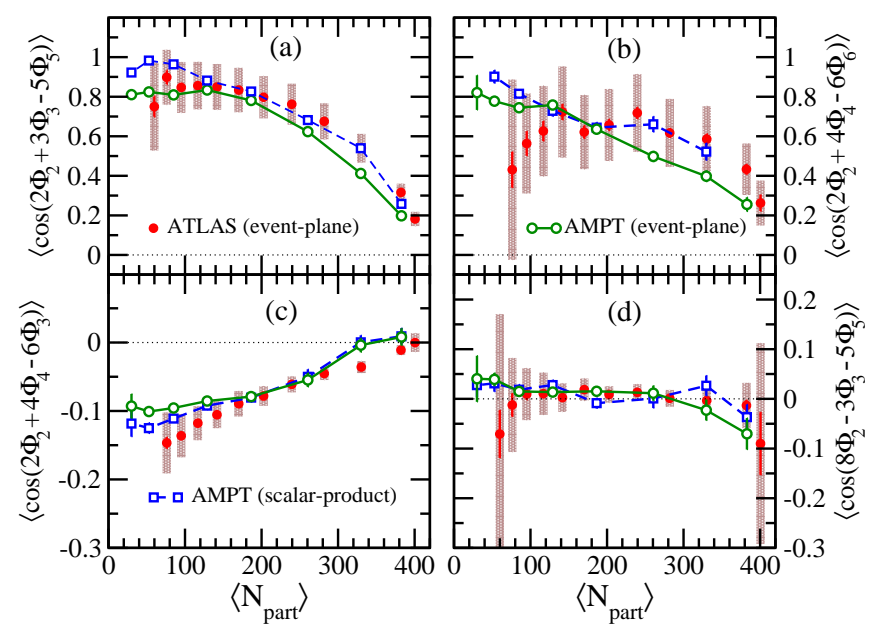

FIG. 2. (Color online) Three-plane correlators. Legend is the same as in Fig. 1. 
The correlations between the second and fourth harmonics (Figs. 1(a)-1(c)) and between the second and sixth harmonics (Fig. 1(e)) are understood [20] as coming mostly from the nonlinear hydrodynamic response, which couples $v_{4}$ to $\left(v_{2}\right)^{2}$ and $v_{6}$ to $\left(v_{2}\right)^{3}$. Their increase from central to peripheral collisions is driven by the increase of $v_{2}$ itself. Similarly, the correlations between harmonics 3 and 6 (Fig. $1(\mathrm{f})$ ) is driven by the coupling between $v_{6}$ and $\left(v_{3}\right)^{2}$ : it decreases slightly as the collision becomes more peripheral, in the same way as $v_{3}$. Finally, the correlation between harmonics 2 and 3 (Fig. 1(d)) is an order of magnitude smaller [13]. Our calculation agrees with data in all cases.

Figure 2 presents AMPT calculations for four threeplane correlators. Again, calculations are in perfect agreement with data. $c\{2,3,-5\}$ and $c\{2,4,-6\}$ (Figs. 2(a) and2(b)) are strong and driven by the nonlinear hydrodynamic response, in the same way as the correlation between harmonics 2 and 4 . The $c\{2,4,-3,-3\}$ correlation has a more subtle origin [17], in the sense that it is not simply generated by the nonlinear response term $v_{4} \propto\left(v_{2}\right)^{2}$, but it is also qualitatively reproduced by event-by-event hydrodynamic calculations [16]. Finally, $c\{2,2,2,2,-3,-5\}$ is compatible with zero.

Figure 3 presents predictions for three new [37] threeplane correlators (Figs. 33(a)-33(c)), as well as three fourplane correlators (Figs. 3(d)-3(f)). The magnitude and centrality dependence of $c\{2,3,3,-4,-4\}$ (Fig. 3(a)) are similar to those of $c\{2,4,-3,-3\}$ (Fig. 2(c)) and likewise, this correlator is not simply generated by the

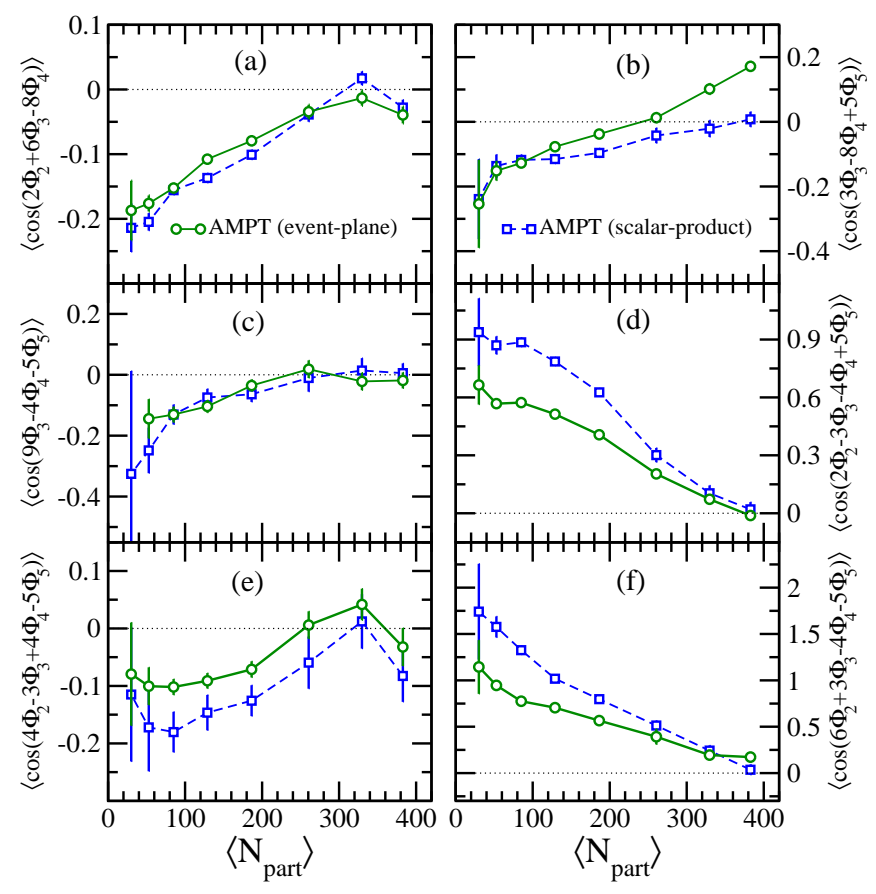

FIG. 3. (Color online) Predictions for new three- and fourplane correlators. Legend is the same as in Fig. 1. $v_{4} \propto\left(v_{2}\right)^{2}$ nonlinear response. Four-plane correlators are much more sensitive to analysis details than two- or three-plane correlators: the difference between the scalarproduct method and the event-plane method is roughly 50\%. Two of these correlators (Figs. 3(d) and 3(f)) are large, which can again be understood as an effect of the nonlinear hydrodynamic response coupling $v_{4}$ to $\left(v_{2}\right)^{2}$ and $v_{5}$ to $v_{2} v_{3}$ [20]. Note that the last four-plane correlator (Fig. [3(f)) is predicted to exceed unity when analyzed using the scalar-product method.

Conclusions. - We have argued that event-plane correlators in heavy-ion collisions can be analyzed with just two symmetric pseudorapidity windows. We have illustrated the validity of our approach by analyzing events simulated within the AMPT model which reproduces for the first time the magnitude and centrality dependence of the measured correlators in $\mathrm{Pb}-\mathrm{Pb}$ collisions at LHC. Much better agreement with data is achieved than in previous hydrodynamic calculations using the event-plane method [16, 17]. This apparent discrepancy between data and hydrodynamic calculations may simply be due to the ambiguity of the event-plane method. It will then be resolved once both experiment and theory switch from the event-plane method to the scalar-product method. We have presented predictions for new correlators, in particular large four-plane correlators, which can be measured in forthcoming analyses. It would be interesting to study the correlators using the procedure presented here in the event-by-event hydrodynamical simulations to ascertain the sensitivity to initial-state models, namely the MonteCarlo Glauber and the color-glass-condensate [42].

This work is funded by CEFIPRA under project 44042. JYO acknowledges support by the European Research Council under the Advanced Investigator Grant ERCAD-267258.

[1] U. W. Heinz and R. Snellings, Ann. Rev. Nucl. Part. Sci. 63, 123 (2013).

[2] C. Gale, S. Jeon and B. Schenke, Int. J. Mod. Phys. A 28, 1340011 (2013).

[3] J. -Y. Ollitrault, Phys. Rev. D 46, 229 (1992).

[4] K. Adcox et al. [PHENIX Collaboration], Phys. Rev. Lett. 89, 212301 (2002).

[5] K. Aamodt et al. [ALICE Collaboration], Phys. Lett. B 708, 249 (2012).

[6] S. Chatrchyan et al. [CMS Collaboration], Eur. Phys. J. C 72, 2012 (2012).

[7] G. Aad et al. [ATLAS Collaboration], Phys. Rev. C 86, 014907 (2012).

[8] S. A. Voloshin, A. M. Poskanzer and R. Snellings, arXiv:0809.2949 [nucl-ex].

[9] B. Alver and G. Roland, Phys. Rev. C 81, 054905 (2010) [Erratum-ibid. C 82, 039903 (2010)].

[10] B. Alver et al. [PHOBOS Collaboration], Phys. Rev. Lett. 98, 242302 (2007). 
[11] A. P. Mishra, R. K. Mohapatra, P. S. Saumia and A. M. Srivastava, Phys. Rev. C 77, 064902 (2008).

[12] R. S. Bhalerao, M. Luzum and J. -Y. Ollitrault, Phys. Rev. C 84, 034910 (2011).

[13] K. Aamodt et al. [ALICE Collaboration], Phys. Rev. Lett. 107, 032301 (2011).

[14] J. Jia [ATLAS Collaboration], Nucl. Phys. A 910-911, 276 (2013).

[15] A. P. S. Yadav and B. D. Wandelt, Phys. Rev. Lett. 100, 181301 (2008).

[16] Z. Qiu and U. Heinz, Phys. Lett. B 717, 261 (2012).

[17] D. Teaney and L. Yan, Nucl. Phys. A 904-905, 365c (2013).

[18] N. Borghini and J. -Y. Ollitrault, Phys. Lett. B 642, 227 (2006).

[19] F. G. Gardim, F. Grassi, M. Luzum and J. -Y. Ollitrault, Phys. Rev. C 85, 024908 (2012).

[20] D. Teaney and L. Yan, Phys. Rev. C 86, 044908 (2012).

[21] C. Adler et al. [STAR Collaboration], Phys. Rev. C 66, 034904 (2002).

[22] M. Luzum and J. -Y. Ollitrault, Phys. Rev. C 87, 044907 (2013).

[23] Z. -W. Lin, C. M. Ko, B. -A. Li, B. Zhang and S. Pal, Phys. Rev. C 72, 064901 (2005).

[24] A. M. Poskanzer and S. A. Voloshin, Phys. Rev. C 58, 1671 (1998).

[25] M. Luzum, Phys. Lett. B 696, 499 (2011).

[26] In practice, one symmetrizes the numerator by exchang- ing $A$ and $B$ to decrease the statistical error.

[27] B. Alver et al., Phys. Rev. C 77, 014906 (2008).

[28] J. -Y. Ollitrault, A. M. Poskanzer and S. A. Voloshin, Phys. Rev. C 80, 014904 (2009).

[29] M. Luzum, J. Phys. G 38, 124026 (2011).

[30] S. Voloshin and Y. Zhang, Z. Phys. C 70, 665 (1996).

[31] F. G. Gardim, F. Grassi, M. Luzum and J. -Y. Ollitrault, Phys. Rev. C 87, 031901(R) (2013).

[32] N. Borghini, P. M. Dinh and J. -Y. Ollitrault, Phys. Rev. C 64, 054901 (2001).

[33] N. Borghini, P. M. Dinh and J. -Y. Ollitrault, Phys. Rev. C 62, 034902 (2000).

[34] R. S. Bhalerao, N. Borghini and J. Y. Ollitrault, Nucl. Phys. A 727, 373 (2003).

[35] A. Adare et al. [PHENIX Collaboration], Phys. Rev. Lett. 105, 062301 (2010).

[36] A. Bilandzic, R. Snellings and S. Voloshin, Phys. Rev. C 83, 044913 (2011).

[37] J. Jia and D. Teaney, arXiv:1205.3585 [nucl-ex].

[38] J. Xu and C. M. Ko, Phys. Rev. C 84, 044907 (2011).

[39] S. Pal and M. Bleicher, Phys. Lett. B 709, 82 (2012); J. Phys. Conf. Ser. 420, 012027 (2013).

[40] W. -T. Deng, X. -N. Wang and R. Xu, Phys. Rev. C 83, 014915 (2011).

[41] L. X. Han, G. L. Ma, Y. G. Ma, X. Z. Cai, J. H. Chen, S. Zhang and C. Zhong, Phys. Rev. C 84, 064907 (2011).

[42] F. Gelis, E. Iancu, J. Jalilian-Marian, and R. Venugopalan, Ann. Rev. Nucl. Part. Sci. 60 (2010) 463. 